\title{
Correlation of Temporomandibular Joint Sound Cases With Direction of Jaw Movement Deviation: A Study of Data Survey at Unsyiah Dental Hospital, Aceh-Indonesia
}

\author{
Liana Rahmayani ${ }^{* 1,3}$, Mustanir ${ }^{2}$, Cut Soraya ${ }^{3}$, Saumi Syahreza ${ }^{4}$ \\ ${ }^{1}$ Graduate School of Mathematics and Applied Science, Universitas Syiah Kuala, \\ Banda Aceh 23111, Indonesia \\ ${ }^{2}$ Department of Chemistry, Faculty of Mathematics and Natural Sciences, Universitas Syiah Kuala, Banda Aceh 23111, \\ Indonesia \\ ${ }^{3}$ Faculty of Dentistry, Universitas Syiah Kuala, Banda Aceh 23111, Indonesia \\ ${ }^{4}$ Department of Chemistry, Faculty of Mathematics and Natural Sciences, Universitas Syiah Kuala, Banda Aceh 23111, \\ Indonesia \\ *Corresponding author.Email: liana.rahmayani@unsyiah.ac.id
}

\begin{abstract}
The temporomandibular joint disorder is a complex and multifactorial problem involving aspects of muscles and joints. Signs and symptoms are joint sounds and deviations during jaw movements such as opening and closing the mouth. Objectives: This study was aimed to show whether there is a correlation between joint sounds that occur with the direction of deviation the jaw movement in Temporomandibular Joint sound cases at Unsyiah Dental Hospital, Aceh. Material and Methods: A cross-sectional study was taken from data of subjects who received prosthodontic treatment at the dental hospital in 2019. From the overall medical record data of 470 prosthodontic treatment subject data, 84 patients were recorded as having temporomandibular joint disorders. However, from 84 data, it turns out that 32 patient data cannot be used and only 52 medical record data can be used. The data collected was analyzed and tested by the Pearson chi-square test and continued with the Fisher's exact (2-sided) test with a significance alpha p-value $<0.05$. Results: The results showed that the most joint sounds in the right and left joint sounds that is a total of 30 (58\%). The highest number of samples is $24(46 \%)$ in the deviation direction to the right. Whereas for the most age range in the age of 46-55 that is equal to 13 subject data (25\%), and it showed that a value of $P$ was 0.378 which means the results were not significant $(P \geq 0.05)$. Conclusion: It can be concluded that there is no correlation between joint sounds with the deviation direction of the jaw movement. The joint sound is not determined by the direction of deviation. There is usually the possibility that right joint sounds can have a left, right, and left-right deviation, as well as other possibilities.
\end{abstract}

Keywords: Deviation, Jaw movement, Joint sound, Temporomandibular disorder

\section{INTRODUCTION}

The temporomandibular joint (TMJ) is a bilateral joint that present in front of the ear, located in the jaw of the human right and left that can move freely within certain limits. The position of the temporomandibular joint in this case, plays an important role in opening and closing movements of the jaw, chewing, and speaking [1].

In certain conditions, the temporomandibular joint can occur disorders or diseases commonly called Temporomandibular Disorder (TMD). In general, TMD causes signs and symptoms that can be felt by patients. Signs and symptoms that are felt tend to be chronic. Common symptoms that complained of are specific pain, head and neck pain, ear buzzing, jaw muscle pain, limited and felt jaw movements and changes in occlusion $[2,3]$. Temporomandibular joint disorder is a complex and multifactorial problem involving aspects of muscles and joints $[4,5]$. Muscles and joints interact simultaneously, but if the function of one or both of them is disturbed, it can cause problems $[3,6,7]$.

The prevalence of TMD is known from several studies, one of them by Karthik et al., Showing a fairly high percentage of $69.4 \%-71.4 \%$ in myofacial pain disorders and $55.5 \%-85.7 \%$ found in disc displacement. This percentage is estimated to increase by $2 \%$ per year [8,9]. According to Chang et al (2018) showed that prevalence of internal derangement of the TMJ in clinic is said to be more than $20 \%$ of the population [10]. An earlier study that characterized patients in TMD and orofacial pain outpatient clinics found that $8 \%$ of cases 
diagnosed only involved TMJ, 73\% only involved masticatory muscles and $19 \%$ had the second type of disorder. According to this study, around 8 million people in Brazil have TMD levels, $90 \%$ of this total are women with age 20 to 45 years old $[11,12,13]$. Then in the 1980s, several authors reported that joint disorders were the most common factor in TMD. However, at present, many authors assert that various dysfunctions can cause pathology in TMJ and masticatory muscles to work separately or together $[14,15]$.

In addition to the TMD symptoms, a common sign is a joint sound and deviation during jaw movement (opening and closing the mouth). Joint sounds are often associated with deviations that occur [16,17]. Causes of muscle irregularities are generally present as large changes in inconsistent gait that are not related to clicking sound $[17,18]$. If clicking exists, this might have poor implications for lateral pterygoid abdominal coordination. If the deviation occurs with the opening range of motion limited to $35 \mathrm{~mm}$ or less and if the opening speed changes the location and pattern of the deviation, the problem is usually intraarticular. Intraarticular deviation is generally associated with joint sounds at the maximum of the opening deviation. This dysfunction can be caused by a disc that is placed correctly with movement restricted by adhesion or by disc movement by reduction [19]. According to Gato et.al (2005) the TMJ on the deviation side showed a smaller condyle and a higher incidence of disk displacement than the nondeviation side and also in the control subjects. However, the clinical symptoms showed no differences between the deviated and nondeviated sides, and no association with disk displacement [20].

The present of muscle dysfunction that causes irregularities can vary depending on the muscles involved. Observing protrusive and laterotrusive movements can help differentiate intra-articular causes from muscle problems. Joint sounds are often associated with deviations that occur. However, no one has been able to prove that the pattern of deviation has anything to do with the sound of the joint itself. Irregularities that are not consistent with ipsilateral joint sounds require sophisticated imaging to explore other pathologies. Therefore this study aims to show whether there really is a correlation between joint sounds and deviations $[21,22]$. Female gender is associated with an increased risk of developing and maintaining myofascial pain. Signs of mandibular instability increase the risk of maintaining TMD signs and symptoms during the observation period [23,24,25]. Widmalm et al study found that deviation in the mandibular path during wide jaw opening and closing can have significant diagnostic interest, especially if associated with TMJ sounds. however, their study was limited to show a correlation between deviation and joint sound, but did not show the direction of the deviation. therefore this study will be something that might be a reference in the diagnosis of TMD because from the available literature no one has discussed it yet [26,27].

\section{MATERIAL AND METHODS}

An analytical descriptive retrospective study with a cross-sectional study approach. Data was collected from medical record data of subjects who received prostodontic treatment at the Unsyiah Dental Hospital, Banda Aceh on May to June 2019. The study was approved by ethics committee of Dental Faculty Universitas Syiah Kuala, and the study was conducted for approximately 2 months. Determination of the sample was using purposive sampling technique, sample selection conducted on the base of consideration of the researchers themselve and according to inclusion criteria. In the Unsyiah Dental Hospital, from the overall medical record data of 470 prosthodontic treatment subject data, 84 patients were recorded as having temporomandibular joint disorders. But from 84 data, it turns out 32 patient data that cannot be used and only 52 medical record data can be used. The inclusion criteria in this study were medical records of patients (male and female) who underwent prosthodontic treatment, from 2015 - 2019, the data taken to patients who lost teeth and had temporomandibular joint disorders (marked by clicking/joint sound and deviation). Exclusion criteria include; medical record data of treatments other than prosthodontics, data of patients with unclear or dubious disorders, and incomplete data.

Collecting data began by searching the data of medical records in computer data base that saving the record of subject information including demographyc data, examination, diagnosis, treatment planning of protheses and the treatment that had done or final treatment. The data obtained was then collected and tabulated data, based on gender and joint disorders in the form of joint sound (on the right, left or both sides of the right and left). It also collected data on the category of deviations that occur (deviations to the right, to the left, or both right and left). Data analysis used Pearson chisquare test and continued with Fisher's Exact (2-sided) test, with alpha p value 0.05 (IBM, Chicago, Version 16). The data analysis was used to determine the correlation between joint sound with the direction of deviation jaw movement.

\section{RESULTS}

The results shown in table 1 can be seen from the total data collected as many as 52 subject data consisting of 24 male and 28 female subject data. The percentage of female gender showed to be greater $(54 \%)$ than the male sample $(46 \%)$. For the most joint sounds in right and left joint sounds that is a total of $30(58 \%)$. Based on table 2 . The highest number of samples is $24(46 \%)$ in the deviation direction to the right. Whereas for the most age range in the age of 46-55 that is equal to 13 subject data (25\%).

Table 4. Pearson chi-square test results and continued with Fisher's Exact show that this case hypothesis, Ho; There is no relationship between joint sounds and 
direction of deviation. Ha; There is a relationship between joint sound and direction of deviation. Decision: The fisher test also shows the Exact Sig. (2-sided) is 0.378 or the probability is $\mathrm{p}$ value $<0.05(0.378>0.05)$ then Hypothesis was rejected. According to analysis, it can be concluded that there is no relationship between joint sound and direction of deviation, or it can be said that joint sound was not determined by the direction of deviation. There is usually the possibility that right joint sounds can have a left, right and left-right deviation, as well as other possibilities.

\section{DISCUSSION}

The position of the temporomandibular joint in this case plays an important role in opening and closing movements of the jaw, chewing, and speaking [1]. In certain conditions in the temporomandibular joint, there can be a disorder or disease commonly called Temporomandibular Disorder (TMD). In general, TMD causes signs and symptoms that can be felt by patients. Signs and symptoms that are felt tend to be chronic. Common symptoms that are complained of are specific pain, head and neck pain, ear buzzing, jaw muscle pain, limited and felt jaw movements and changes in occlusion [2]. Temporomandibular joint disorders are complex and multifactorial problems involving aspects of muscles and joints. Muscles and joints interact simultaneously, but if the function of one or both of them is disturbed, it can cause problems $[1,2]$.

In this study, research data were taken from subjects who had joint sounds with a variety of variations. Sound on the right side, left side and both sides right and left. In addition, data from the direction of jaw movement were taken. According to table 1, it was found that there were 18 more female subjects $(56 \%)$ than 14 male subjects (44\%). According to Susanna et al, female sex is associated with an increased risk of developing and maintaining myofascial pain. Also in Laplanche et.al study found that TMD problem are widespread, affecting $90 \%$ of general population at one life stage or another, especially 20-40 years old women. Signs of mandibular instability increase the risk of maintaining TMD signs and symptoms during the observation period [24,25]. As for joint sounds, the highest percentage is those that experience sounds on both the right and left sides of $56 \%$. Based on table 3 . The most visible TMD disorders in the form of joint sounds in the age range $17-25$ years and $36-45$ years, amounting to $28 \%$. This age is an age that is susceptible to interference, because it is considered productive age compared to old age, in which the joints have begun to adapt to the disorders that occur $[23,26,27]$.

Subjects Known in addition to symptoms of TMD, a common sign is a joint sound and deviation during jaw movement (opening and closing the mouth). Some opinions state joint sounds are often associated with the occurrence of deviations that occur. But in this study the results found no significant correlation between the occurrence of joint sounds with the direction of deviation. In other words joint sound on one side has nothing to do with the direction of the deviation, which means that the right side sound is not necessarily the direction of deviation to the left, or otherwise [27,28]. The Betsy et al research showed., Which states that muscle dysfunction that causes irregularities can vary depending on the muscles involved [21,29]. Observing protrusive and laterotrusive movements can help differentiate intra-articular causes from muscle problems. Irregularities that are not consistent with ipsilateral joint sounds require sophisticated imaging to explore other pathologies $[20,25,30]$.

\section{CONCLUSION}

According to the results of this study, it can be concluded that there is no significant correlation between temporomandibular joint sound and the presence of joint sound that occurs. it can be said that joint sound is not determined by the direction of the deviation. There may be a possibility that the sound of the right joint can have a left deviation, a right or left-deviation, and other possibilities.

\section{REFERENCES}

[1] Bigaton DR., Dibai Filho AV, de Souza Costa AC, Packer AC, de Castro EM. Accuracy and Reliability of Infrared Thermography in The Diagnosis of Arthralgia in Women With Temporomandibular Disorder. Journal of Manipulative and Physiological Therapeutics 2013;36:253-8.

[2] Graff-Radford SB, Abbott JJ. Temporomandibular Disorders and Headache. Oral Maxillofacial Surg Clin N Am 2016;28:335-49.

[3] Okeson J. Management of Temporomandibular Disorders And Occlusion. 8th ed. CV. Mosby; 2019.

[4] Kapos, F. P., Look, J. O., Zhang, L., Hodges, J. S. \& Schiffman, E. L. Predictors of long-term temporomandibular disorder pain intensity: An 8year cohort study. J. Oral Facial Pain Headache2018; 32: 113-122.

[5] Murphy MK, MacBarb RF, Wong ME, Athanasiou KA. Temporo-mandibular disorders: a reviewof etiology, clinical manage-ment, and tissue engineering strategies. Int J Oral MaxillofacImplants 2013;28:e393-414.

[6] Dawson P. Functional occlusion from TMJ to smile design. St. Louis: Mosby, Inc.2008.

[7] Rodrigues-Bigaton D, Dibai-Filho AV, Packer AC, de Souza Costa AC, de Castro EM. Accuracy of Two Forms of Infrared Image Analysis of The Masticatory Muscles in The Diagnosis of Myogenous Temporomandibular Disorder. Journal of Bodywork \& Movement Therapies 2014;18:49-55.

[8] Karthik R, Fathima MH, Saravanan C, Vivek N, Priyadarsini P, Ashwath B. Assessing prevalence of temporomandibular disorders among university students: A questionnaire study. Journal of 
international society of preventive and community dentistry 2017;7:24-29.

[9] Martinez CC, Espinosa de santillana IA, Wintergerstlavin AM, Tostado FL. Prevalence of temporomandibular disorders according to RDC/TMD, in patients of asub-urban community of puebla, mexico. Revista Colombia de Investigacion en Odontologia 2013; 1-9.

[10]Chang CL, Wang DH, Yang MC, Hsu WE, Hsu ML. Functional disorders of the temporomandibular joints: Internal derangement of the temporomandibular joint. The Kaohsiung Journal of Medical Sciences 2018; 34(4):223-230.

[11]Progiante, P. et al. Prevalence of Temporomandibular Disorders in an Adult Brazilian Community Population Using the Research Diagnostic Criteria (Axes I and II) for Temporomandibular Disorders (The Maringá Study). Int. J. Prosthodont 2015; 28: 600-609.

[12] Gauer RL, Semidey MJ. Diagnosis and Treatment of Temporomandibular Disorders. Am Fam Physician 2015;91:378-86.

[13] Kohler AA, Hugoson A, Magnusson T. Prevalence of symptoms indicative of temporomandibular disorders in adults:cross-sectionalepidemiological investigations coveringtwodecades. Acta Odontologica Scandinavica 2012;70:213-223.

[14] Kalladka M, Quek S, Heir G, Eliav E, Mupparapu M,Viswanath A. Temporomandibular joint osteoarthritis: diag-nosis and long-term conservative management: a topic re-view. J Indian Prosthodont Soc 2014;14:6-15.

[15]Edefonti V, Bravi F, Cioffi I, Capuozzo R, Ammendola L, Abate G, Decarli A, Ferraroni M, Farella M, Michelotti A. Chronic pain and weather conditions in patients suffering from temporomandibular disorders: a pilot study. A. Community Dent Oral Epidemiol 2012 Feb;40(1):56-64.

[16] Carlsson GE, Magnusson T, Guimarães AS. Management of temporomandibular disorders in the general dental practice. Chicago: Quintessence; 2006.

[17] De Melo Ju'nior PC, Aroucha JMCNL, Arnaud M, De Souza Lima MG, Gomes SGF, Ximenes R, et.al. Prevalence of TMD and level of chronic pain in a group of Brazilian adolescents. Plus One 2019;14:213.

[18] Meloto, C. B. et al. Clinical predictors of persistent temporomandibular disorder in people with first onset temporomandibular disorder: A prospective case-control study. J. Am. Dent. Assoc 2019; 150: 572- 581.e10.

[19] Estela T. Temporomandibular joint sounds and disc dislocations incidence after orotracheal intubation. Clinical, Cosmetic and Investigational Dentistry 2009;1:71-3.

[20] Goto TK, Nishida S, Nakayama E, Nakamura Y, Sakai S, Yabuuchi H, et.al. Correlation of mandibular deviation with temporomandibular joint
MR dimensions, MR disk position, and clinical symptoms. Oral Surgery, Oral Medicine, Oral Pathology, Oral Radiology, and Endodontology 2005;100: 743-9.

[21]Betsy Mitchel DC, Cathy Cummins DC, Ron LeFebvre DC . Temporomandibular Joint Disorders (TMD): A Clinical Assessment, university of western states; 2015. P. 1-6.

[22] Shepard. Headache. Orofacial Pain: A Guide for the Headache Physician. 2013.

[23] Marklund S, Wanman A. Risk factors associated with incidence and persistence of signs and symptoms of temporomandibular disorders. Acta Odontologica Scandinavica 2010; 68: 289-299.

[24] Laplanche O, Ehrmann E, Pedeutour P, Duminil G. TMD Clinical Diagnostic Classification (Temporo Mandibular Disorder). J Dentofacial Anom Orthod 2012;15:202.

[25] Petersson A. What you can and cannot see in TMJ imaging - an overview related to the RDC/TMD diagnostic system. Journal of Oral Rehabilitation 2010;37:771-778.

[26]Zhang S, Zhang Z, Yu W, Ren Y, Ye D, Wang L, et.al. Analysis of The Correlation Between Morphology and Kinematics of Anteriorly Displaced TMJ Discs Using Cine-MRI and ARCUSdigma Systems. The Open Dentisty Journal 2018;12:904-15.

[27] Wildmalm SE, Dong Y. Li B, Lin M, Fan L, Deny SM. Unbalanced Lateral Mandibular Deviation Associated With TMJ Sound as a sign in TMJ Disc Dysfunction Diagnosis. Journal of Oral Rehabilitation 2016;43:911-20.

[28] Sener S, Akgnlu F. Correlation Between The Condyle Position and Intra-Extraarticular Clinical Finding of Temporomandibular Dysfunction. European Journal of Dentistry 2011;5:354-60. List T, Jensen RH. Temporomandibular disorders: Old ideas and new concepts. Cephalalgia 2017;37(7), 692-704.

[29] Miller VE, Poole C, Golightly Y, Barrett D, Chen DG, Ohrbach R, Slade GD. Characteristics associated with high-impact pain in people with TMD: a cross-sectional study. The Journal of Pain 2018; 20(3): 288-300. 